\title{
Swaying oscillons in the signum-Gordon model
}

\author{
H. Arodź $\tilde{z}^{a}$ and $\quad$ Z. Świerczyński ${ }^{b}$ \\ ${ }^{a}$ Institute of Physics, Jagiellonian University, Cracow, Poland \\ ${ }^{b}$ Institute of Computer Science and Computer Methods, \\ Pedagogical University, Cracow, Poland
}

\begin{abstract}
We present a new class of oscillons in the (1+1)-dimensional signumGordon model. The oscillons periodically move to and fro in the space. They have finite total energy, finite size, and are strictly periodic in time. The corresponding solutions of the scalar field equation are explicitly constructed from the second order polynomials in the time and position coordinates.
\end{abstract}

PACS: 05.45.-a, 03.50.Kk, 11.10.Lm 


\section{Introduction}

Scalar fields play essential role in many branches of physics, from cosmology to condensed matter physics to particle physics - there is an unremitting interest in models of self-interacting scalar fields. The rich variety of such models includes some that have been studied only recently, e.g., the so called K-fields with a nonstandard kinetic part [1], or models with a non-smooth V-shaped self-interaction [2]. The signum-Gordon model considered in the present paper is probably the simplest example from the latter class. The pertinent field potential has the form $U(\varphi)=g|\varphi|$, where $g>0$ is a coupling constant and $|\varphi|$ is the modulus of the real scalar field $\varphi$. Such potential is V-shaped with the sharp minimum at the vacuum field $\varphi=0$. Models of this kind were discovered while playing with the well-known classical systems of harmonically coupled pendulums in order to illustrate the phenomenon of spontaneous symmetry breaking and topological defects [3]. Subsequent investigations have revealed that the V-shaped form of the potential has very interesting consequences for the dynamics of the scalar field. One of them is the existence of strictly periodic oscillons [4]. The motivation, various results and further references for the V-shaped self-interaction can be found in [2, 3, 4].

The present note is a follow-up to the paper [4]. The oscillons described in that paper did not move in space (apart from the trivial uniform motion obtained by applying Lorentz boosts). Rather unexpectedly, we have found that there exist also oscillons that periodically move to and fro in the space with arbitrary constant velocity $\pm v$, where $0<|v| \leq 1$. For the oscillons presented in [4] $v=0$. The new oscillons appear naturally when the particular solution reported in [4] is put in the framework of polynomial solutions of the signum-Gordon equation.

Comparing with other oscillons discussed in literature [5], several differences should be pointed out. First, our oscillons are strictly periodic in time, in particular they do not emit any radiation. Second, they have strictly finite size because the field assumes the vacuum value at a finite distance exactly. Third, they have relatively simple, explicitly given form composed of several linear and quadratic functions of the time $t$ and the spatial coordinate $x$.

The swaying oscillon reminds the wobbling kink in the $\varphi^{4}$ model [6]. However, one should note that the wobbling kink is an excitation of static kink, while all the swaying oscillons are degenerate in energy, and moreover there is no static oscillon - even for the presented in [4] non-swaying one the field oscillates in time.

The plan of our paper is as follows. Section 2 is devoted to a preliminary 
discussion of the signum-Gordon equation and of its solutions. The swaying oscillons are presented in Section 3. Section 4 contains the conclusion.

\section{Preliminaries}

The Lagrangian of the signum-Gordon model (s-G) has the form

$$
L=\frac{1}{2}\left(\partial_{t} \varphi \partial_{t} \varphi-\partial_{x} \varphi \partial_{x} \varphi\right)-g|\varphi|,
$$

where $\varphi$ is a real scalar field, $t, x$ are time and position coordinates in the twodimensional Minkowski space-time $M$. For convenience, $t, x, \varphi, g$ are dimensionless - this can be achieved by redefinitions of the physical position, time, field and the coupling constant (multiplication by constants of appropriate dimensions). The signum-Gordon equation

$$
\partial_{t}^{2} \varphi-\partial_{x}^{2} \varphi+\operatorname{sign}(\varphi(x, t))=0
$$

is the Euler-Lagrange equation corresponding to Lagrangian (1) (from now on we put $g=1)$. The sign function $\operatorname{sign}(\varphi)$ has the values \pm 1 for $\varphi \neq 0$ and $\operatorname{sign}(0)=0$. The simplest way to obtain Eq. (2) from Lagrangian (1) is first to regularize the field potential $U(\varphi)=|\varphi|$, e.g., $U(\varphi)=\sqrt{\epsilon^{2}+\varphi^{2}}$ or $U(\varphi)=$ $\epsilon \ln (\cosh (\varphi / \epsilon))$, and to take the limit $\epsilon \rightarrow 0_{+}$in the Euler-Lagrange equation obtained from the regularized Lagrangian. Direct computation of the variation of the action $S=\int d t d x L$ is more subtle because of the $|\varphi|$ term, but it gives the signum-Gordon equation (2) too.

The 1.h.s. of Eq. (2) is not continuous with respect to $\varphi$. Because such equations are not very common in field theory, let us briefly comment on the related mathematical aspects. First, it is clear that in general one should expect non smooth solutions: the value of at least one of the second derivatives $\partial_{t}^{2} \varphi, \partial_{x}^{2} \varphi$ has to jump when the function $\operatorname{sign}(\varphi)$ changes its value. Second, the use of the stationary action principle implies that in general we consider so called weak solutions of the Euler-Lagrange equation, [7]. For the weak ones it is sufficient that

$$
\delta S=\int_{M} d t d x\left(\frac{\partial L}{\partial \phi} \delta \phi(x, t)+\frac{\partial L}{\partial\left(\partial_{\mu} \phi\right)} \partial_{\mu} \delta \phi(x, t)\right)=0
$$

for all test functions $\delta \phi(x, t)$ from a certain class (typically one uses the $D(M)$ class of smooth functions on $M$ with compact support). This condition is equivalent to $\int_{M} d t d x \mathcal{E} \delta \varphi=0$, where $\mathcal{E}=\partial L / \partial \phi-\partial_{\mu}\left(\partial L / \partial\left(\partial_{\mu} \varphi\right)\right)$, only if the 
derivative $\partial_{\mu}\left(\partial L / \partial\left(\partial_{\mu} \varphi\right)\right)$ exists for a given probed function $\varphi(x, t)$. Then the Euler-Lagrange equation $\mathcal{E}=0$, in our case the signum-Gordon Eq. (2), has to be satisfied at almost all points $(x, t)$ in the two-dimensional space-time $M$, but not necessarily at all points as it would be the case with strong solutions. Of course, the set of weak solutions contains the strong ones as a subset.

In the case of signum-Gordon equation the weak solutions that are not strong are ubiquitous. For instance, $\varphi_{0}=x^{2} / 2$ is a smooth static solution of Eq. (2) in the weak sense, but not in the strong sense. The point is that $\partial_{t}^{2} \varphi_{0}-\partial_{x}^{2} \varphi_{0}+\operatorname{sign}\left(\varphi_{0}\right)=$ 0 everywhere in $M$ except the line $x=0$ in $M$. On this line $\partial_{t}^{2} \varphi_{0}-\partial_{x}^{2} \varphi_{0}+$ $\operatorname{sign}\left(\varphi_{0}\right)=-1$ because $\partial_{x}^{2} \varphi_{0}=1, \operatorname{sign}(0)=0$. Nevertheless,

$$
\int_{M} d t d x\left[\partial_{t}^{2} \varphi_{0}-\partial_{x}^{2} \varphi_{0}+\operatorname{sign}\left(\varphi_{0}\right)\right] \delta \phi(x, t)=0
$$

for arbitrary test function $\delta \phi$.

In general, physically relevant are the weak solutions. To see this, consider the following simple example from classical mechanics of a point particle on a plane with Cartesian coordinates $(x, y)$. The particle is free except when it crosses the $y$-axis, where it is subjected to a finite constant force $\vec{F}_{0}$ parallel to the $y$ axis. Thus, the force $\vec{F}=0$ at all points $(x, y)$ with $x \neq 0$, and $\vec{F}=\vec{F}_{0}$ when $x=0$. It is clear that integrating the Newton's equation $d \vec{p} / d t=\vec{F}$ we obtain $\vec{p}=$ const even if the trajectory crosses the $y$-axis. The physical reason is that the finite force $\vec{F}_{0}$ acts on the particle only during infinitesimally short time when the particle is exactly on the $y$-axis, hence it is not able to perturb the free motion. Such trajectories are the weak solutions of the Newton's equation (now the test functions are denoted as $\delta \vec{r}(t)$ and we integrate over $t$ ). On the other hand, the trajectories which do not intersect the $y$-axis are solutions in the strong sense. Notice that such Newton's equation is not equivalent to the free equation, in which $\vec{F}=0$ everywhere, because our particle is accelerated if it moves along the $y$-axis.

Coming back to the signum-Gordon model, in the case the field $\varphi$ is constant in the space Eq. (2) acquires the form of one dimensional Newton's equation $\ddot{\varphi}(t)=-\operatorname{sign}(\varphi)$ that describes nonlinear oscillations around $\varphi=0$. Notice that there is no linear regime even for arbitrarily small values of $\varphi$. Newton's equation of this kind appears in the elementary problem of a ball vertically bouncing from a floor in a constant gravitational field (the elevation above the floor is given by $|\varphi|)[2]$. Many examples of oscillatory systems from classical mechanics that do not have the linear small amplitude regime can be found in [8].

Because the function $\operatorname{sign}(\varphi)$ is piece-wise constant, it is natural first to solve Eq. (2) in the regions in which $\varphi$ has a constant sign. For instance, if $\varphi<0$, Eq. 
(2) acquires the form

$$
\partial_{t}^{2} \varphi-\partial_{x}^{2} \varphi-1=0
$$

The oscillon solutions are constructed from second order polynomials in $x, t$. The most general second order polynomial that obeys Eq. (3) has the form

$$
\varphi_{2}(x, t)=a_{0} x^{2}+a_{1} t x+\left(a_{0}+\frac{1}{2}\right) t^{2}+b_{0} x+b_{1} t+c_{0}
$$

where $a_{0}, a_{1}, b_{0}, b_{1}, c_{0}$ are constants (beware that they are not completely arbitrary because of the condition $\varphi_{2}<0$ ). It is rather exceptional feature of the signumGordon equation that non-trivial and interesting solutions can be constructed from such simple elementary functions. Note that the class of functions of the form (4) is invariant with respect to Lorentz boosts, space-time translations, and the reflections $x \rightarrow-x, t \rightarrow-t$. It contains the static solutions of the form

$$
\varphi_{s}=-\frac{1}{2}\left(x-b_{0}\right)^{2}+c_{0}+\frac{1}{2} b_{0}^{2}
$$

where $c_{0}+b_{0}^{2} / 2<0$ in order to keep $\varphi_{s}<0$.

The oscillons are constructed by patching together several such polynomial solutions. The patching conditions have the standard form: the field $\varphi$ is continuous all over $M$, also the derivatives $\partial_{t} \varphi, \partial_{x} \varphi$ are continuous function of $x, t$ except perhaps at the border line between two patches. If the border line is a (segment of) characteristic line ( $x= \pm t+$ const) for the signum-Gordon equation, the derivative in the direction perpendicular to that line does not have to be continuous - a finite jump is allowed.

\section{The swaying oscillons}

Hint that new oscillons may exist comes from the following procedure for constructing periodic solutions of the signum-Gordon equation. Let $\varphi_{-}(x, t)$ be a solution of Eq. (3) negative for all $t$ from an open interval $(0, T), T>0$, and such that

$$
\varphi_{-}(x, 0)=0=\varphi_{-}(x, T) .
$$

It is clear that the function $\varphi_{+}$defined by

$$
\varphi_{+}(x, t)=-\varphi_{-}(x,-t)
$$


is a positive solution of the equation $\partial_{t}^{2} \varphi-\partial_{x}^{2} \varphi+1=0$ for all $t \in(-T, 0)$. The functions $\varphi_{-}, \varphi_{+}$as well as their time derivatives match each other at the time $t=0$ :

$$
\begin{gathered}
\varphi_{+}(x, 0)=0=\varphi_{-}(x, 0), \\
\lim _{t \rightarrow 0_{-}} \partial_{t} \varphi_{+}(x, t)=-\lim _{t \rightarrow 0_{-}} \partial_{t} \varphi_{-}(x,-t)=\lim _{s \rightarrow 0_{+}} \partial_{s} \varphi_{-}(x, s),
\end{gathered}
$$

where $s$ stands for $-t$, and $t \in(-T, 0)$. The crucial observation is that also $\varphi_{+}(x,-T), \varphi_{-}(x, T)$ match each other:

$$
\begin{gathered}
\varphi_{+}(x,-T)=0=\varphi_{-}(x, T), \\
\lim _{t \rightarrow-T_{+}} \partial_{t} \varphi_{+}(x, t)=\lim _{s \rightarrow T_{-}} \partial_{s} \varphi_{-}(x, s) .
\end{gathered}
$$

Therefore, we may extend our partial solutions $\varphi_{ \pm}$to all times $t \geq T$ and $t \leq-T$ just by applying time translations (by multiples of $\pm T$ ) to $\varphi_{ \pm}$. In this way we obtain periodic solutions of the signum-Gordon equation (2) with the period equal to $2 T$, provided that there exists $\varphi_{-}(x, t)$ with the properties specified above. It turns out that a class of the solutions $\varphi_{-}(x, t)$ with the desired properties can be constructed by patching together several solutions of the form (4). Also the trivial solution $\varphi=0$ is involved. The schematic picture of such 'patchwork' for the swaying oscillon is presented in Fig. 1.

Note that so far we have not made any assumption about the behavior of $\varphi_{-}$ at large $|x|$. In [4] certain restrictive boundary conditions were imposed right at the start of calculations and they forced the oscillons to stay still. The method adopted in the present note is radically different from the one used in [4] - in that paper the main tool was d'Alembert formula for solutions with given initial data.

In order to ensure finiteness of the total energy we assume that $\varphi_{-}(x, t)=0$ outside a certain compact region. This is related to the general observation that in the case of models with the V-shaped potential there are no exponential or long range tails. The field reaches its vacuum value rather abruptly, the tails have a parabolic shape and a strictly finite length [2]. Thus, our first task is to find the polynomials of the form (4) which match the trivial solution $\varphi=0$. The matching conditions imposed on a line $x(t)$ in $M$ can be written in the form

$$
\varphi_{2}(x(t), t)=0,\left.\quad \partial_{x} \varphi_{2}(x, t)\right|_{x=x(t)}=0 .
$$

They give the following two equations

$$
a_{0} x^{2}(t)+a_{1} t x(t)+\left(a_{0}+\frac{1}{2}\right) t^{2}+b_{0} x(t)+b_{1} t+c_{0}=0, \quad 2 a_{0} x(t)+a_{1} t+b_{0}=0 .
$$


Simple calculations show that the solution $x(t)$ exists only if $a_{0} \neq 0$, and then

$$
x(t)=v t+x_{0}, \quad \varphi_{2}(x, t)=-\frac{(x-x(t))^{2}}{2\left(1-v^{2}\right)},
$$

where $v=-a_{1} /\left(2 a_{0}\right), \quad x_{0}=-b_{0} /\left(2 a_{0}\right)$, and $v^{2}<1$ in order to satisfy the condition $\varphi_{2}<0$. Here we have assumed that $x(t)$ does not coincide with a characteristic line.

Thus we have found that the boundary of our oscillon has to move with the constant velocity $v$, and close to the boundary the field has the parabolic shape (as expected). Note that $\varphi$ given by formula (8) coincides with the Lorentz boosted and translated in the space the static solution

$$
\varphi_{s}=\left\{\begin{array}{cc}
0 & x \leq 0 \\
-\frac{x^{2}}{2} & x>0
\end{array}\right.
$$

(but the swaying oscillon is not the Lorentz boosted oscillon of [4]).

The structure of the solution $\varphi_{-}$is shown in Fig. 1, in which the support of $\varphi_{-}$ for the swaying oscillon of unit length and vanishing total momentum is depicted. The period of this oscillon is equal to its spatial size, i.e., to 1. As discussed in [4], we may use the symmetries of the signum-Gordon equation, such as Poincaré or scaling transformations in order to obtain more general oscillons. The interior of the parallelogram is divided into seven sectors $a \div f$ by the four characteristic lines drawn from its corners. Each sector has different causal neighborhood. For instance, the field in the triangular sector $c$ is completely determined by Cauchy data on the segment $[1 / 2+v / 2,1]$ of the $x$ axis; the sector $e$ is controlled by Cauchy data in the future, i.e., on the segment $[1 / 2,1+v / 2]$ of the $t=1 / 2$ line which lies in the future of the sector $e$; the sectors $a$ and $d$ are controlled by the boundaries of the oscillon, etc. The parallelogram shown in Fig. 1 has the height equal to one half of its length. In this case the characteristic lines drawn from the lower (upper) corners meet at a point lying on the upper (lower) edge.

In the case of the non-swaying oscillon presented in [4] we have $v=0$ and a rectangle in Fig. 1. The parallelogram is the simplest deformation of that rectangle consistent with the conditions $\varphi_{-}(x, 0)=0=\varphi_{-}\left(x, \frac{1}{2}\right)$, and with the fact that the both sides of the oscillon have to move with a constant velocity, as has been shown above. Such generalization - the parallelogram instead of the rectangle is very suggestive in the 'patchwork approach' adopted in the present paper, but it is not obvious at all in the based on the d'Alembert formula approach used in [4]. Let us also note that a Lorentz boost of the oscillon considered in [4] gives 
a uniform rectilinear motion, and not the swaying one. Moreover, it deforms the rectangle into a hyperbolically rotated parallelogram with the upper and bottom sides not parallel to the $x$-axis.

The building blocks of $\varphi_{-}$are denoted as $\varphi_{a}, \ldots, \varphi_{g}$ after the sectors of the parallelogram. The fields $\varphi_{a}, \varphi_{d}$ in the sectors $a$ and $d$ have the form (8) with $x(t)=v t$ or $x(t)=v t+1$, respectively, i.e.,

$$
\varphi_{a}=-\frac{(x-v t)^{2}}{2\left(1-v^{2}\right)}, \quad \varphi_{d}=-\frac{(x-v t-1)^{2}}{2\left(1-v^{2}\right)} .
$$

In the regions $x \leq v t$ and $x \geq v t+1$, i.e. on both sides of the parallelogram, the field has the vacuum value $\varphi=0$.

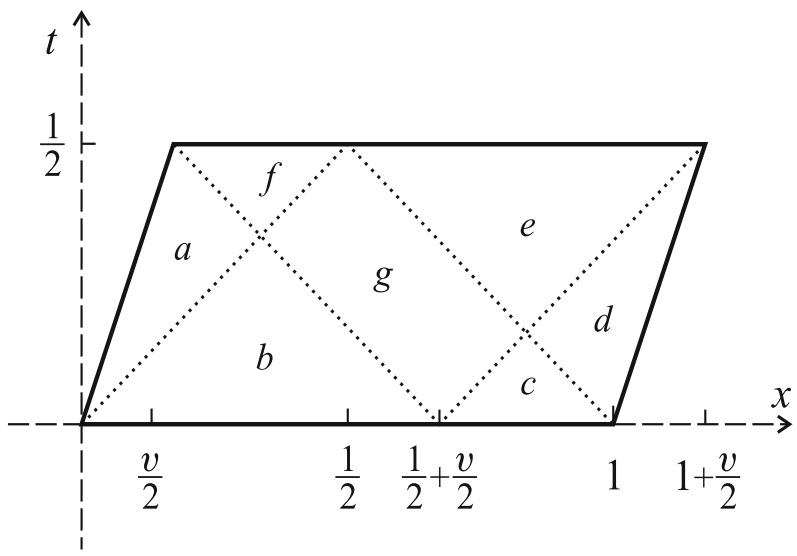

Figure 1: The support of the solution $\varphi_{-}(x, t)$. The field $\varphi_{-}(x, t)$ vanishes on the continuous lines that form the boundary of the parallelogram. In each sector $a \div f$ the function $\varphi_{-}$is given by a different formula. The matching conditions that relate the functions in neighboring sectors are imposed along the dotted lines. These four lines are characteristic lines of the signum-Gordon equation. They have the slopes \pm 1 .

The fields $\varphi_{b}, \varphi_{c}, \varphi_{e}, \varphi_{f}$ are determined by imposing on the solution (4) the condition $\varphi_{2}=0$ on the lines $t=0$ or $t=1 / 2$, and the conditions of matching with $\varphi_{a}$ or $\varphi_{d}$ on the characteristic lines. As the example let us determine $\varphi_{b}$. The condition $\varphi_{2}(x, 0)=0$ gives $a_{0}=b_{0}=c_{0}=0$. Next, $\varphi_{2}=t^{2} / 2+a_{1} t x+b_{1} t$ is compared to $\varphi_{a}$ on the part of the characteristic line $x=t$ with $t \in(0,1 / 4+v / 4)$ :

$$
\frac{t^{2}}{2}+a_{1} t^{2}+b_{1} t=-\frac{1-v}{2(1+v)} t^{2} .
$$


Therefore, $b_{1}=0, a_{1}=-1 /(1+v)$, and $\varphi_{b}=t^{2} / 2-t x /(1+v)$. Similar calculations give $\varphi_{c}, \varphi_{e}, \varphi_{f}$. Finally, we compute $\varphi_{g}$ by comparing $\varphi_{2}$ to $\varphi_{b}, \varphi_{c}, \varphi_{e}, \varphi_{f}$ along the four characteristic lines that form the boundary of the sector $g$. The results have the following form:

$$
\begin{gathered}
\varphi_{b}=\frac{t^{2}}{2}-\frac{t x}{1+v}, \quad \varphi_{c}=\frac{t^{2}}{2}+\frac{t(x-1)}{1-v}, \\
\varphi_{e}=\frac{1}{2}\left(t-\frac{1}{2}\right)\left(\frac{1}{2}+t+\frac{1-2 x}{1+v}\right), \\
\varphi_{f}=\frac{1}{2}\left(t-\frac{1}{2}\right)\left(\frac{1}{2}+t+\frac{2 x-1}{1-v}\right), \\
\varphi_{g}=\frac{(v x+t)^{2}}{2\left(1-v^{2}\right)}+\frac{x^{2}+t^{2}}{2}+\frac{1+v}{8(1-v)}-\frac{x+t}{2(1-v)} .
\end{gathered}
$$

All these functions are negative inside their domains. The shape of the swaying oscillon is depicted in Fig. 2.

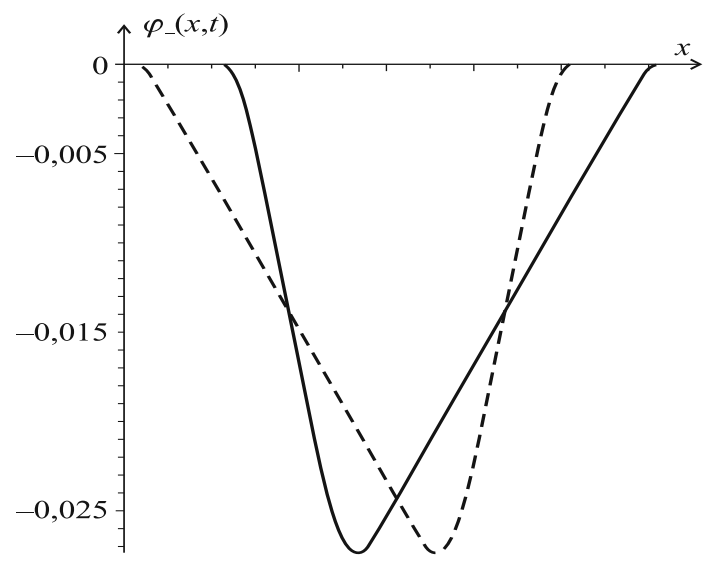

Figure 2: The shape of the swaying oscillon at the times $t=1 / 8$ (the dashed line) and $t=3 / 8$ (the continuous line). The velocity of the swaying motion $v=1 / 2$.

The evolution of our oscillon is described by the function $\varphi_{-}(x, t)$ in the time interval $[0,1 / 2]$, and by $\varphi_{+}(x, t)$, formula (7), for $t \in[-1 / 2,0]$. In particular, the field $\varphi_{+}$at the boundaries of the oscillon has the form

$$
\varphi_{+, a}(x, t)=\frac{(x+v t)^{2}}{2\left(1-v^{2}\right)}, \quad \varphi_{+, d}(x, t)=\frac{(x+v t-1)^{2}}{2\left(1-v^{2}\right)} .
$$


We see that now the boundaries of the oscillon move with the velocity $-v$. The world-sheet of the oscillon is depicted in Fig. 3. Note that at the times $t=k / 2, k$ - integer, when the sharp turns take place, the field $\varphi$ vanishes everywhere.

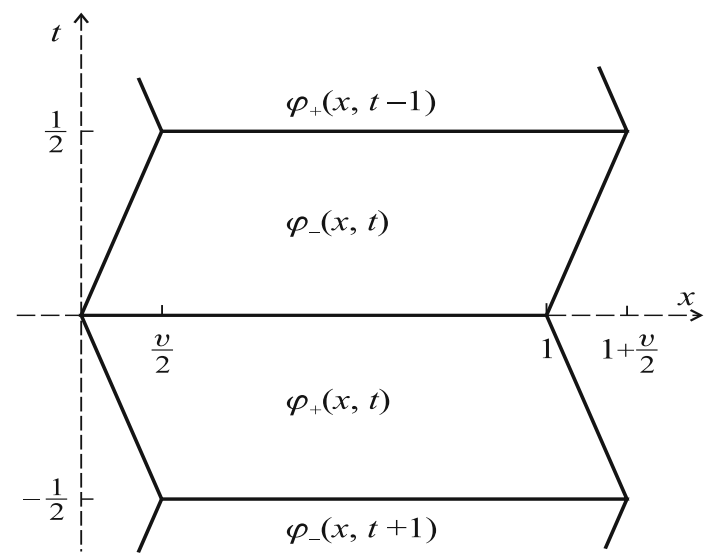

Figure 3: The world-sheet of the swaying oscillon. In the interior of the parallelograms $\varphi_{+}>0$ and $\varphi_{-}<0$, whereas on their boundaries (the thick continuous lines) $\varphi_{ \pm}=0$.

In the case $x(t)$ is a characteristic line we have $x(t)=v t+x_{0}$, where $|v|=$ 1. There is just one matching condition $\varphi_{2}(x(t), t)=0$. Solving it we obtain relations between the constant coefficients present in $\varphi_{2}$, and finally

$$
\varphi_{2}(x, t)=(x-x(t))\left(a_{0}(x-x(t))-\frac{1}{2} x(t)+\left(\frac{1}{2}+2 a_{0}\right) x_{0}+b_{0}\right)
$$

(in the region where $\varphi_{2}(x, t)<0$ ). Next steps are similar to those described above, but the situation is much simpler. In particular, when $v=1$, the left and right hand sides of the parallelogram in Fig. 1 coincide with characteristic lines. Therefore, the sectors $a, f, c, d, g$ are absent. The remaining sectors $b, e$ meet at the line $x=1-t$. The corresponding functions $\varphi_{b}, \varphi_{e}$ are given by formulas (10), (11) with $v=1$, and they correctly match each other on that line.

The total energy $E$ and momentum $P$ of the oscillon can easily be calculated from formulas

$$
E=\frac{1}{2} \int_{-\infty}^{\infty} d x\left[\left(\partial_{t} \varphi\right)^{2}+\left(\partial_{x} \varphi\right)^{2}\right]+\int_{-\infty}^{\infty} d x|\varphi|, \quad P=-\int_{-\infty}^{\infty} d x \partial_{t} \varphi \partial_{x} \varphi
$$


considered at the time $t=0$ when $\varphi=0=\partial_{x} \varphi$. We see that $P=0$, in spite of the swaying motion of the oscillon. This can be understood if we regard the swaying oscillon as a nonlinear bound state of the basic oscillon, that is the one with $v=0$, with a wave packet traveling along the basic oscillon. If the swaying oscillon has $P=0$, the nonzero momentum of the wave packet is compensated by the momentum related with the motion of the basic oscillon. The wave packet bounces from the boundaries of the basic oscillon and does not leave its interior. Then the basic oscillon has to move accordingly in order to keep $P=0$.

In order to compute the total energy we need $\left.\partial_{t} \varphi\right|_{t=0}$. Formulas (9), (10) give $\left.\partial_{t} \varphi_{b}\right|_{t=0}=-x /(1+v)$ for $x \in[0,(1+v) / 2]$, and $\left.\partial_{t} \varphi_{c}\right|_{t=0}=(x-1) /(1-v)$ for $x \in[(1+v) / 2,1]$. In the case $v= \pm 1$ the part with $\varphi_{c}$ is absent. Simple integration gives $E=1 / 24$. Thus the total energy does not depend on $v$-all our swaying oscillons have the same energy. We have not found any explanation for such a degeneracy. One may suspect that there exists a hidden symmetry. Note that it would be sufficient if it works only in the subspace of the polynomial solutions $\varphi_{2}$, not necessarily on the level of Lagrangian or action. The bound state interpretation offers the following picture. The basic oscillon set in motion would have an energy larger that its rest energy equal to 1/24. Apparently, the binding energy compensates the kinetic energy of the basic oscillon as well as the energy of the bouncing wave packet, so that the total energy remains equal to $1 / 24$.

\section{Conclusion}

We have shown that oscillons in the (1+1)-dimensional signum-Gordon model can periodically move to and fro in the space (the x-line) with a constant speed $v$ from the interval $[0,1]$. The amplitude of such swaying motion is equal to $v l / 2$, where $l$ is the length of the oscillon. The pertinent analytic solutions of the field equation have been constructed from the second order polynomials in $t$ and $x$.

The present paper is a follow-up to [4], and the remarks and comments given there apply also to the swaying oscillons. Our new findings contribute to the already substantial evidence that the models of the signum-Gordon type have rather amazing properties. In particular, it is quite surprising that one can find simple, explicit solutions that describe very nontrivial objects like the oscillons, or $Q$-balls [9], and this happens in spite of the unpleasant $\operatorname{sign}(\varphi)$ form of the nonlinear term in the field equation. 


\section{References}

[1] C. Armendariz-Picon, T. Damour and V. F. Mukhanov, Phys. Lett. B 458, 2009 (1999); C. Adam, P. Klimas, J. Sanchez-Guillen and A. Wereszczyński, J. Math. Phys. 50: 102303 (2009).

[2] H. Arodź, P. Klimas and T. Tyranowski, Acta Phys. Pol. B 36, 3861 (2005); Phys. Rev. E 73, 046609 (2006).

[3] H. Arodź, Acta Phys. Pol. B 33, 1241 (2002); ibidem 35, 625 (2004).

[4] H. Arodź, P. Klimas and T. Tyranowski, Phys. Rev. D 77, 047701 (2008).

[5] See, e.g., I. L. Bogolyubskii and V. G. Makhankov, JETP Lett. 24, 12 (1976); M. Gleiser, Phys. Lett. B 600, 126 (2004); M. Hindmarsh and P. Salmi, Phys. Rev. D 74, 105005 (2006); G. Fodor, P. Forgacs, P. Grandclément and I. Rácz, Phys. Rev. D 74, 124003 (2006); M. Gleiser and J. Thorarinson, Phys. Rev. D 76, 041701(R) (2007); M. Gleiser and D. Sicilia, Phys. Rev. Lett. 101, 011602 (2008).

[6] See, e.g., I. V. Barashenkov and O. F. Oxtoby, Phys. Rev. E 80, 026608 (2009); O. F. Oxtoby and I. V. Barashenkov, Theor. Math. Phys. 159, 863 (2009).

[7] See. e.g., R. D. Richtmyer, Principles of Advanced Mathematical Physics. Springer-Verlag, New York-Heidelberg-Berlin, 1978. Section 17.3. L. C. Evans, Partial Differential Equations. American Math. Society, 1998.

[8] P. Mohazzabi, Am. J. Phys. 72, 492 (2004).

[9] H. Arodź and J. Lis, Phys. Rev. D 77, 107702 (2008). 